\title{
Motion Learning Based on Bayesian Program Learning
}

\author{
Meng-Zhen CHENG, Quan-Hua TANG, Long-Jun HUANG \\ School of Software, Jiangxi Normal University, Jiangxi, China \\ e-mail: 1538801696@qq.com,593913588@qq.com,huanglong98614@163.com
}

\begin{abstract}
The concept of virtual human has been highly anticipated since the 1980s. By using computer technology, Huma n motion simulation could generate authentic visual effect, which could cheat human eyes visually. Bayesian Program Learning train one or few motion data,generate new motion data by decomposing and combining. And the generated motion will be more realistic and natural than the traditional one.In this paper, Motion learning based on Bayesian program learning allows us to quickly generate new motion data, reduce workload, improve work efficiency, reduce the cost of motion capture, and improve the reusability of data.
\end{abstract}

\section{Introduction}

In 2009, the special effects of the movie-Avatar caused a burst of viewing boom. The vivid Na'vi people, all kinds of creatures, magnificent scenes in the movie all show the magic of computer animation technology. Among them, the movements of Na'vi people refers to the movements of actors and completes by using the late processing simulation through computer technology. All of these are done through computer graphics technology, the usage of this technology has a lot of work, such as Toy Story Mania, Cars, Spiderman and so on. These films have many effects, such as toys and cars could walking, jumping and moving, express emotions and so on. With the de-velopment of computer animation, it is a challenge that how to become more natural to liquidate the body posture and varieties of movements. The $\mathrm{Na}$ 'vi is a virtual pers-on, human motion simulation is a technology that compu-ter could build a virtual person to simulate human behavi-or in a natural world.

Machine learning[1] is the way your computer using experience to optimize system performance, it is an important branch of artificial intelligence. Machine learning is the study of how to make the computer simulation process of human learning knowledge, innovation and reconstruction of the existing knowledge in order to improve the system's ability to deal with problems, we also call it optimize performance. Artificial neural network and Bayesian learning are the focus of research in machine learning, but also the two field of in-depth study.

Bayesian Learning was derived from the proof of a special case of the British mathematician, Thomas, in 1763, in the case of a book on the theorem of the author of the book of letters by the author of the book. Bayesian learning is one of the main algorithms to solve machine learning problems. Bayesian learning provides a solution for the study of uncertain expression, while but the real world and mankind itself have their own uncertain problems, artificial intelligence solves how to answer these uncertainties, thus the Bayesian learning show the advantages compared to other algorithms. Algorithm of Bayesian learning compared to other learning algorithms, better able to ignore the impact of the error data, and can handle incomplete data.[2-4]

Bayesian program learning[5] been presented in the study of human level concept learning based on Bayesian programming learning by Brenden M. Lake et al from three schools in North America,breaking the barriers that the traditional machine learning to thousands of examples to train.It marks a big step in the field of machine learning.In this paper, based on the Bayesian program learning method, only one or few examples can be used to make the virtual person learn the abstract concept of motion, so as to achieve the effect of simulation, and even generate additional examples.

\section{Related Notion}

\subsection{Human model}

Human motion simulation is the use of computer simulation of virtual human behavior in the natural world of technology, in order to make the virtual human action realistic, it is necessary to fully simulate the human bones, muscles and other elements. Therefore, there are many models to simulate human motion, such as surface model, solid model, 
multilevel model and rod like model (skeletal model). No matter what kind of model is the study of the geometric characteristics of the human body and the use of geometric characteristics to establish the model of the response characteristics. Because of this study is using the method of Bayesian learning process of learning and human motion simulation, and the main elements to support human motion is the bones and muscles are attached to the bones, with the skeletal motion and change the form of the human motion, learning is the learning of the movement of the human skeleton, so this article uses stick to show the human motion model[6].

Stick Model (Skeleton Model). The rod model is composed of joints and stick to a simple graphical representation of the human body model, through joint torsion and mobile graphics bar to show the movement of the human body, its complexity to be decided by the joint. This model of data storage is small, easy to edit, simple a variety of body posture, but because of rod like graphics, only describe the skeletal structure, this model can represent human motion when reverse action, realistic vision of the weak.[7]

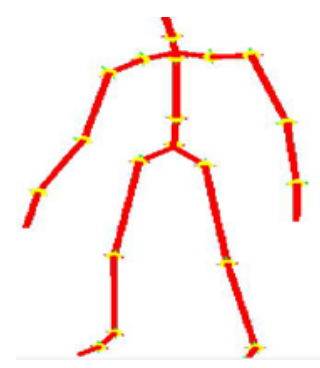

Figure 1. stick model (skeleton model)

\section{2 human motion data}

The human body is composed of 206 bones, 639 muscle tissue composition, the structure is very complex, and the movement of the human body is actually human bones and muscles, so the human motion description is very complicated, a slight movement is likely to involve multiple bone and muscle tissue movement. How to simulate the movement of the human body more realistic and smoothly becomes a great challenge to the research of virtual human. Motion capture technology is the process of recording the human motion by the sensor, and converting it into the data information of human motion. Because this method can effectively capture the real human motion data, mapping the motion of virtual human is a real human motion, so the effect of real human motion is very similar, and this technique can simulate complex movements.

This study is based on the motion capture, however, due to the high cost of motion capture data, this paper use Biovision company to carry on the research of human motion data to research the BVH file format.Finally, the generated data is stored in $\mathrm{BVH}$ format.

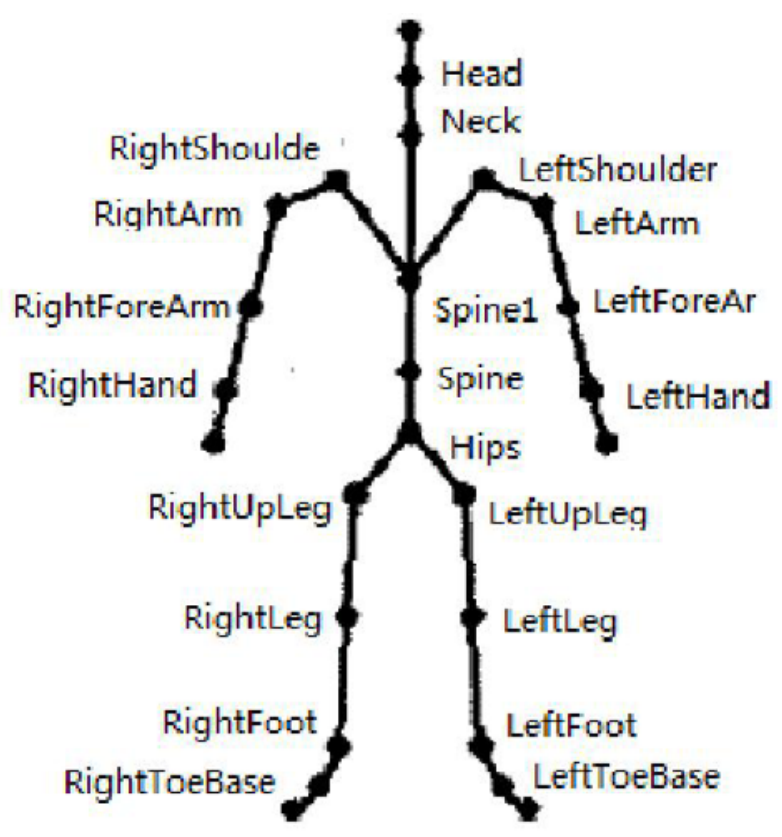

Figure2. Human skeleton structure model

\subsection{Bayesian program learning}

In 2004, Lebeltel et al. Proposed the concept of Bayesian program for solving the problem of robot uncertainty for the first time. This concept is based on Bayesian reasoning and Bayesian learning, the aim is to calculate the $\mathrm{P}(\mathrm{Y} \mid \mathrm{X}$, Data, Knowledge), is the use of a simple target concept ( things to do), the motion data and motion data have been inferred to infer the motion data of the next step, we get the target concept of motion data.The core idea of the Bayesian program is to convert the target into a number of conditional probability distributions function using the Bayesian's law and the chain rule.The Bayesian program can reaffirm many of the classical probability models such as Bayesian Network (BN), Hidden Markov Model (HMM), and so on. The main goal of the Bayesian program is to effectively control the robot to complete a complex action, greatly improving the performance of the robot work for the robot to contribute more intelligence to a force. And after the in-depth study of experts and scholars in each country, people on the Bayesian program of artificial intelligence learning methods and further research results.

In 2015, Brenden.M.Lake et al. Proposed a Bayesian learning procedure based on the concept of human level-Bayesian Program learning, the robot uses 50 character sample automatic analysis reasoning wrote a number of new characters, in which the robot learn to draw inferences and the like.And these new characters passed the Turing test, people can not distinguish people write and the robot write from the many characters.Previous machine learning methods require a large amount of sample data to support, but people does not need to learn a lot of learning samples, people can learn from a few or even a sample of 
knowledge.Brenden and others believe that a man has such a strong learning ability is because the human is able to analyze things, extract feature rich and representative from a single object, and use these features to construct other examples,then,this knowledge is acquired.Bayesian learning to simplify the concept of rich things, and uses probabilistic semantics to deal with the noise and capture the abstraction of the accidental structure, and then construct a procedure under the Bayesian criterion. In this paper, we will study the simulation of human motion by means of Bayesian programming,make a virtual person able to simulate a new action through one or more actions..

\section{Motion learning}

\subsection{Technology Roadmap}

Motion learning based on Bayesian program learning using the method of Bayesian program learning to learn the human motion data, and generate new motion data.The Bayesian program learning framework enables virtual humans to learn abstract concepts from a few or even a sample, as humans do, and to learn other instances independently from these abstract concepts. The following is the sequence diagram of motion learning.

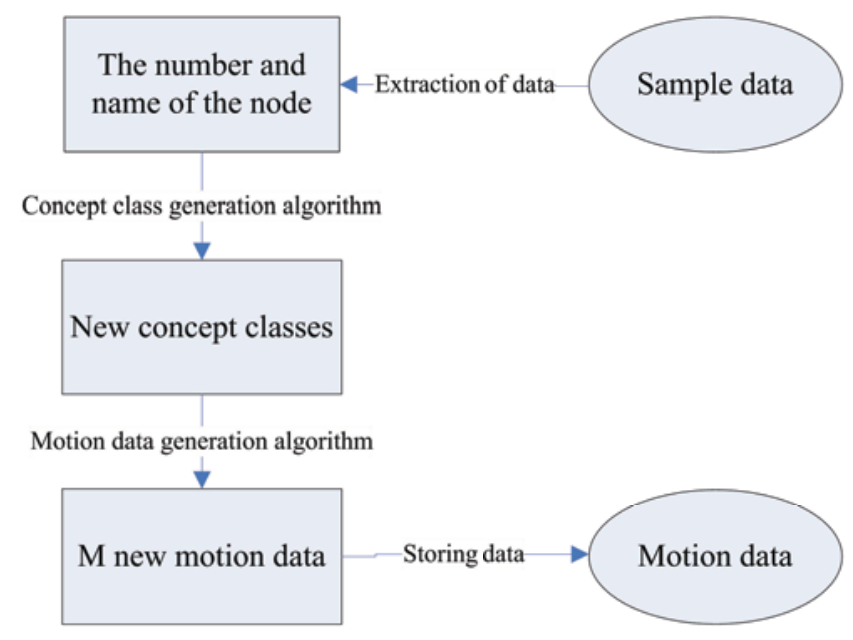

Figure3.Sequence diagram of motor learning

\subsection{Concept class generation algorithm}

In the motion learning based on Bayesian program learning, the input motion data is analyzed and reorganized to obtain the new concept class $\Psi$.After analysis, virtual human will be divided into 5 parts, namely the left hand, right hand, the left leg, right leg and spine; according to the storage of human skeletal structure in the BVH file that the first joint point of each part is the parent node of the lower joint. Therefore, it is necessary to study the relationship between the first joint and the root node of each component.
$\Psi=\{\mathrm{H}, \mathrm{S}, \mathrm{R}\}$ is composed of the location of the root node, the 5 parts $\mathrm{S}=\{\mathrm{S} 1, \mathrm{~S} 2, \ldots, \mathrm{S} 5\}$, and the location relationship between them and the root node $R=\{R 1$, $\mathrm{R} 2, \ldots, \mathrm{R} 5\}$.

$$
P(\psi)=P(H) \prod_{i=1}^{5} P\left(S_{i}\right) P\left(R_{i} \mid S_{1}, \ldots, S_{i-1}\right)
$$

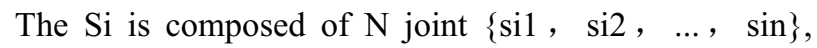
$\mathrm{sij}=\{x i j, y i j, z i j\}, z i j$ is the name of the joint; yij is the maximum rotation angle of each joint point, and with the parent node location close relationship; xij is the joint point around the $\mathrm{X}$ axis and $\mathrm{Y}$ axis, $\mathrm{Z}$ axis angle.

$$
P(S i)=\prod_{j=1}^{n} P\left(x_{i j} \mid z_{i j}\right) P\left(y_{i j} \mid z_{i j}\right)
$$

Among them, P ( xij|zij) accord with Gauss distribution, P (yij|zij) accord with Gamma distribution.

Algorithm description:

Function GenerateType:

\{

for $i=1$ to 5 do //Analysis of virtual human 5 parts

Get the joint $\mathrm{n}$ and $\mathrm{z}$ for the first i parts

Get parts $\mathrm{Si}$

Through the empirical analysis of the sample data, calculate the relationship Ri between the part $\mathrm{Si}$ and the root node

end for

New concept class $\Psi=\{\mathrm{H}, \mathrm{S}, \mathrm{R}\}$

$$
\text { \} }
$$

After the calculation of the concept class generation algorithm, a new concept class $\Psi$ is obtained. This concept class is a study of the existing concepts, abstracting the existing sample data, and obtaining the most empirical and probabilistic calculation. The appropriate one, will be extracted, the combination, and obtained.But this is only the extraction of abstract concepts, and did not generate new sports data, the following will be a detailed description of the generation of motion data.

\subsection{Motion data generation algorithm}

Get a new concept class, and then generate a new sports data is simple, and everyone's movement is basically similar,the difference is that each person's small habits will lead to different forms of motion and amplitude.For the human body movement simulation is the joint position and rotation angle of the different.As long as the new concept of the class to add a number of motion variables, that is, the joint point of the position and rotation angle to add some noise, it can produce a number of new motion, that is, a number of motion data frames. 
Therefore, after getting a new concept class, the new motion data is generated by the following steps:

The initial position of each part of the human body is sampled in the motion data.Li(m) is obtained by calculating the joint distribution of parts $\mathrm{S} 1(\mathrm{~m}), \ldots, \mathrm{Si}-1(\mathrm{~m})$ and $\mathrm{Ri}(\mathrm{m})$ that obtained by the sampling of the position relationship between the first joint point of each part and the root node.

$$
L_{i}{ }^{(m)}=P\left(L_{i}{ }^{(m)} \mid R_{i}{ }^{(m)}, S_{1}{ }^{(m)}, \ldots, S_{i-1}{ }^{(m)}\right)
$$

Among them, Ri (m) through clustering the Ri of each part in the sample, to add noise points to the new concept of the class Ri.

二、Scaling the rotation angle of each joint point in the component,the rotation angle of each joint node in the sample data and the position relation of the parent node are clustered respectively, adding the noise points to the angle of rotation xij and the location relationship with the parent node yij.

$$
\begin{aligned}
& x_{i j}^{(m)}=P\left(x_{i j}{ }^{(m)} \mid x_{i j}\right) \\
& y_{i j}{ }^{(m)}=P\left(y_{i j}{ }^{(m)} \mid y_{i j}\right)
\end{aligned}
$$

三、Through the above two steps, $\operatorname{Li}(\mathrm{m}), \mathrm{xij}(\mathrm{m})$ and yij(m)constitute a deterministic function of $\mathrm{F}$, each $\mathrm{Si}(\mathrm{m})$ is a deterministic function. Thus,obtained $\mathrm{m}$ new component $\mathrm{S}(\mathrm{m})=\{\mathrm{S} 1(\mathrm{~m}), \mathrm{S} 2(\mathrm{~m}), \ldots, \mathrm{S} 5(\mathrm{~m})\}$ with different trajectories and shapes.

$$
\mathrm{S}_{\mathrm{i}}{ }^{(\mathrm{m})}=f\left(L_{\mathrm{i}}{ }^{(\mathrm{m})}, x_{\mathrm{ij}}{ }^{(\mathrm{m})}, y_{\mathrm{ij}}{ }^{(\mathrm{m})}\right)
$$

\author{
Algorithm description: \\ Function GenerateData: \\ \{ \\ for $\mathrm{i}=1$ to $5 \mathrm{do}$ \\ Calculate the distance with $\mathrm{Ri}$ and save it to $\mathrm{r}[]$ \\ end for \\ Divide the smallest of $\mathrm{r}[\mathrm{]}$ to $\mathrm{Ri}(\mathrm{m})$ \\ Determine $\mathrm{m}$ starting positions $\mathrm{Li}(\mathrm{m})$ \\ for $\mathrm{j}=1$ to $\mathrm{n}$ do $/ / \mathrm{n}$ is the total number of joints
}

Calculate the nearest $x i j(m)$ with xij distance,calculate the nearest yij(m)with yij distance end for

Get the data of each component $\mathrm{Si}(\mathrm{m})$ end for \}

NDatas get new motion data NDatas

\section{Experimental and Evaluation}

The experimental data of the BVH format file as input, the motion data in each frame for analysis, analysis of the components as shown in the following figure:

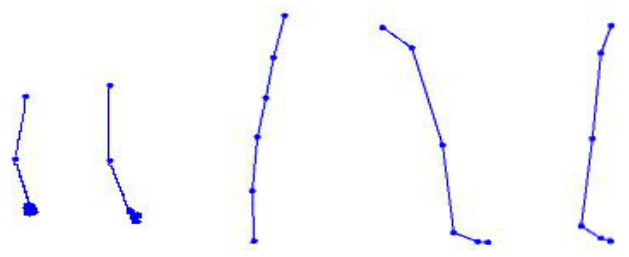

Figure4.Each part

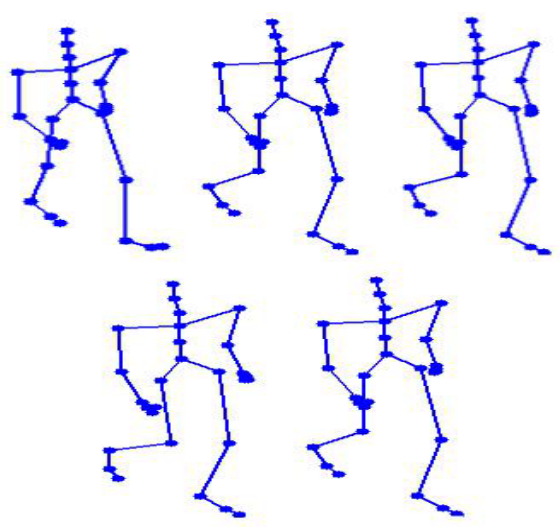

Figure5. New motion

Using Matlab to map the acquired motion data to a virtual person, the virtual human action is obtained. First, the action is compared with the real movement of human beings, and the virtual human movement is evaluated from the perspective of the authenticity of the virtual human movement and the nature.Virtual human action is more natural, in line with the rules of human m-ovement, more real.Then, the new motion data obtained by the experiment was fitted with the bvh format data captured by Biovision's motion, and the similarity was $79.64 \%$.From the above two points, we can know that the algorithm gen-erated by the algorithm is more consistent with the real human movement data, but in the futureresearch there is still much room for improvement.

\section{Conclusion}

This article provides an effective way to learn about sports. In this paper, our study includes two algorithms: concept class generation algorithm, motion data generation algorithm. After research, the generated data and motion capture data similarity is higher, but in the future work study will be further research..

\section{Conclusion}


This work was supported by the Science and Technology Research Project of Jiangxi Provincial Department of Education (GJJ13232).

\section{References}

[1] Tom M. Mitchell .Machine Learning [M].Machinery Industry Press, 2004.

[2] Lebeltel O,Bessière P,Diard J,et al.Bayesian robot programming $[\mathrm{J}]$.Autonomous Robots, 2004,16(1): 4979.

[3] Diard J,Bessiere P,Mazer E.A survey of probabilistic models using the bayesian programming methodology as a unifying framework[C].2003.

[4] Russell S,Norvig P. Artificial intelligence: a modern approach[J].1995.
[5] Brenden M.Lake,Ruslan Salakhutdinov,Joshua B.Tenenbaum.Human-level concept learning through probabilistic program induction. Science,2015,11: 1332-1338.

[6] Fua P. Plänkers R,Thalmann D.Realistic Human Body Modeling[C].In Fifth International Symp- osium on the 3-D Analysis of Human Movement, Chattanooga, TN, 1998, 5(1): 360 372.

[7] Thalmann D.Human Modelling and Animation[R].Eurographics'93 State-of-the-Art Reports, Chapter 7,2004, 18(2): 49-54. 